\title{
Complex-Impedance Dipole Antennas as RFID-Enabled Ice Monitors
}

\author{
Mahmoud Wagih and Junjie Shi \\ School of Electronics and Computer Science, University of Southampton, Southampton, SO17 1BJ, United Kingdom \\ \{Mahmoud.Wagih.Mohamed, Junjie.Shi\}@ soton.ac.uk
}

\begin{abstract}
RFID tags can operate in a wide variety of environments enabling battery-free pervasive sensing. In this paper, timevarying impedance measurements are presented to evaluate the sensitivity of inductive-matched dipole antennas as ice thawing monitors. Relative Signal Strength (RSS) measurements of an encapsulated RFID tags based on a commercial IC are presented showing that the tags can be read for up to 45 minutes inside $9 \mathrm{~mm}$-thick thawing ice. Furthermore, the relationship between the ice thickness and the RSS of the ice-loaded RFID tags. The experimental results show that inductive-fed dipole antennas are highly suited for RFID-based monitoring of ice formation and thawing for smart city and remote sensing applications.
\end{abstract}

\section{INTRODUCTION}

RFID sensing has attracted significant research interest for a variety of application [1]. RFID sensor tags exhibit a change in their phase and relative signal strength (RSS) in response to a stimulant, enabling wireless battery-free sensing based on low-cost tags using commercial RFID ICs and readers. Dipole antennas with an inductive loop, for matching the RFID IC's impedance without the need for lumped components, are widely considered a standard in RFID antenna design [2]. Furthermore, robust RFID antennas have been demonstrated for applications where the tag may be exposed to repetitive bending or washing, such as wearables [3], [4].

Monitoring the formation of ice and its water content or thawing process is of great interest for various applications. For example, detecting the formation of ice in smart cities can be utilized to prevent water pipes for bursting in winter [5], as well as avoiding slips and workplace injuries [6]. Furthermore, identifying the composition of snow basins, i.e. the ratio of ice, snow, and water, enables RFID-based avalanche monitoring and prediction [7]. A two-port RF resonator were proposed for as ice formation and thawing detection [8]. In [5], we investigated the performance of a robust conformable RFID tag, [3], as a wireless ice detection sensor [5], demonstrating an RSS change over $10 \mathrm{~dB}$ in the presence of ice. Nevertheless, detailed analysis of the antenna's impedance was not performed. In addition, the antenna's response during the state transitions, e.g. thawing, was not investigated.

In this paper, we use time-varying impedance and RSS measurements to evaluate the performance of an RFID inductive dipole antenna as an ice sensor, showing high sensitivity to thawing ice. Section II presents impedance measurements of the dipole antenna and Section III presents the performance of the ice-monitoring RFID tag.

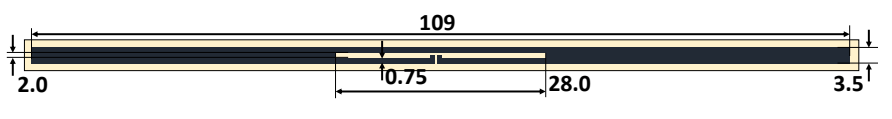

Fig. 1. Layout and dimensions of the RFID antenna-under-test.

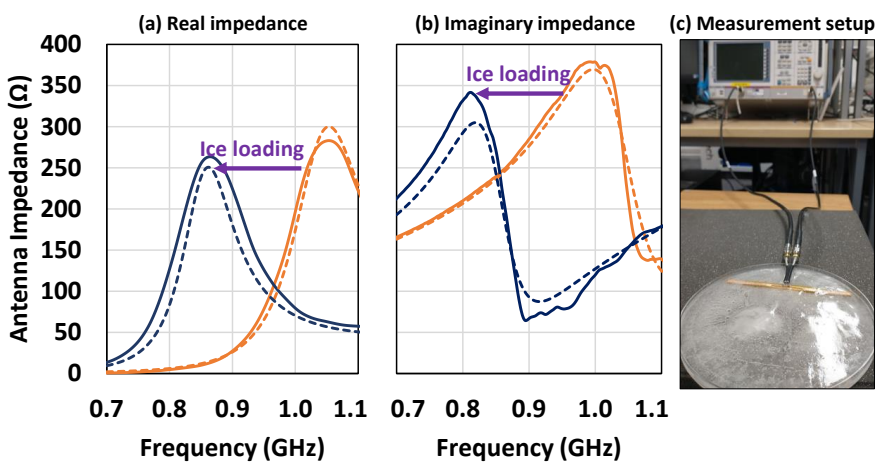

Fig. 2. Simulated (dashed) and measured (solid) antenna impedance in the presence and absence of $7 \mathrm{~mm}$ ice.

\section{ANTENNA IMPEDANCE CHARACTERIZATION}

The antenna used in this work is an inductive-fed compact dipole, whose dimensions are shown in Fig. II. with the detailed design described in [3]. The antennas were fabricated on a polyimide $\left(\epsilon_{r}=3.2, \tan \delta=0.02\right)$ substrate that is approximately $50 \mu \mathrm{m}$-thick. The input impedance of the antenna was measured using a balanced coaxial jig in space (on a foam holder) and when loaded with $7 \mathrm{~mm}$-thick ice. Fig. 22 a) and (b) show the simulated (CST Microwave Studio) and measured real and imaginary input impedance of the unloaded and iceloaded antenna, measured using test setup in Fig. 2(c).

As observed in Fig. 2, the ice results in a resonance shift of approximately $200 \mathrm{MHz}$, improving the impedance matching of the tags in the $868 \mathrm{MHz}$ license-free band. This results in an increase in the RSS of the tags compared to their unloaded response, which can be measured wirelessly to detect the presence of ice. As ice has a low $\tan \delta$ of 0.0009 , it does not reduce the antenna's radiation efficiency.

On the other hand, water is more lossy in the microwave spectrum, with a $\tan \delta$ of 0.044 at $915 \mathrm{MHz}$ at $25^{\circ} \mathrm{C}$ [9], and has a higher relative permittivity of 78.1. Therefore, as the ice melts at room temperature $\left(24^{\circ} \mathrm{C}\right)$, the antenna's resonance 


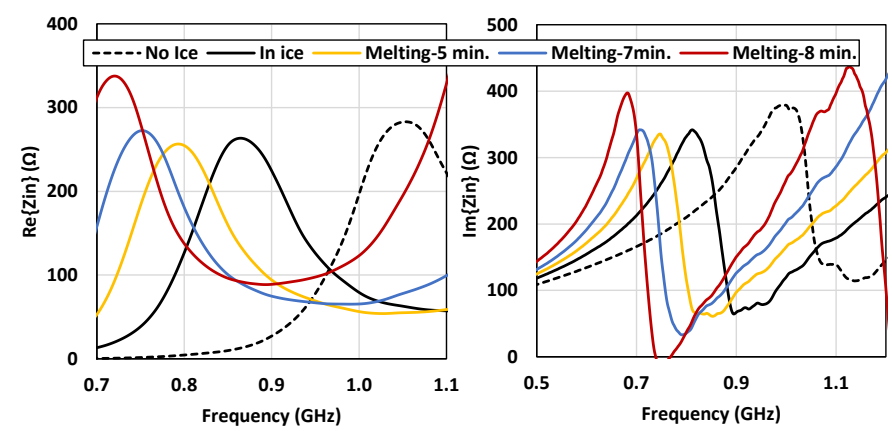

Fig. 3. Simulated and measured antenna impedance in the presence and absence of $7 \mathrm{~mm}$ ice.

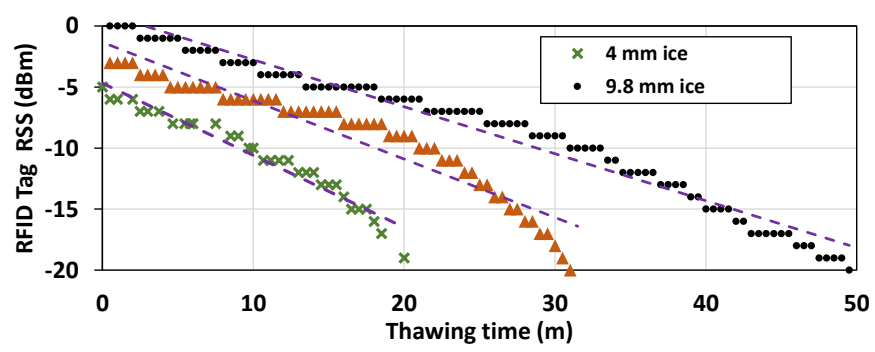

Fig. 4. Measured tag RSS as a function of the thickness of the ice sheet after 10 minutes of thawing.

is expected to shift significantly, and the radiation efficiency will degrade due to the high $\tan \delta$. Fig. 2 shows the measured impedance of the dipole antenna as the $7 \mathrm{~mm}$-thick ice disc melts. In 8 minutes of thawing, it can be observed that not only does the resonance shift by over $400 \mathrm{MHz}, \Re\{Z\}$ increases indicating an increase in the loss resistance, both translating to a decrease in the RSS of the RFID tags, evaluated in the next section.

\section{RFID TAG ICE-MONITORING PERFORMANCE}

The antennas were connected to an NXP UCODE IC with a $-21 \mathrm{dBm}$ threshold and 12-j292 $\Omega$ input impedance at 868 MHz. A $33 \mathrm{dBm}$ EIRP circularly polarized reader (Zebra RFD8500) was used to interrogate the ice-loaded and unloaded tags, at room temperature, to monitor the effect of thawing on their RSS. Fig. 4 shows the normalized RSS of three tags, placed at $5 \mathrm{~m}$ from the reader, for different ice thicknesses.

As the ice melts, the water content increases detuning the tags and reducing their radiation efficiency and gain. This is attributed to the high real and imaginary permittivity of water $\left(\epsilon_{r}=78, \tan \delta=0.2\right)$ compared to ice $\left(\epsilon_{r}=3.2, \tan \delta=0.0009\right)$ [10], and is in line with the observed change in the antenna's measured impedance in Fig. 3. Beyond a certain point, the water content fully detunes the RFID tags making them unreadable, e.g. minute 20 for the $4 \mathrm{~mm}$-thick ice.

In Fig. 4 it can be observed that the RSS varies with the thickness of the ice, with a thicker ice accumulation taking longer to melt and resulting in a longer reading time. Fig. 5 shows the RSS of the thawing ice-loaded tags at 10 minutes,

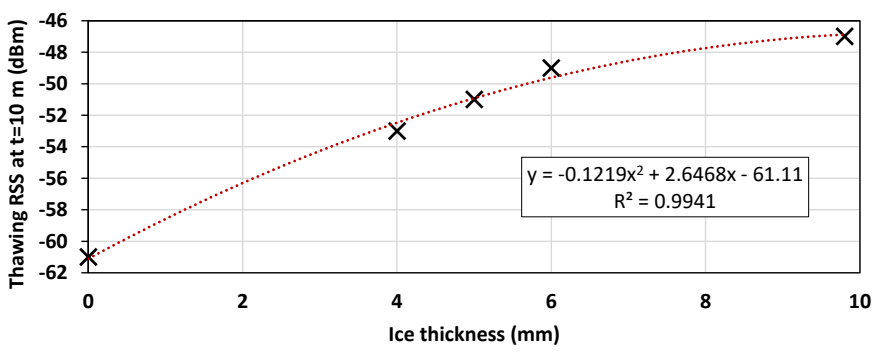

Fig. 5. Measured tag RSS as a function of the thickness of the ice sheet after 10 minutes of thawing.

along with an unloaded tag (thickness $=0$ ). From the measured and fitted RSS, it can be observed that the RSS of the RFID tags can be used to evaluate the thickness of ice during melting. Therefore, RFID tags can be used in applications such as remote environmental sensing to detect the melting of snow basins [7].

\section{CONCLUSION}

In this paper, the performance of complex- $Z$ dipole antennas as ice monitors was investigated. It was found that water and ice can be easily distinguished through the RSS of ice-loaded RFID tags, owing to the permittivity different between water and ice. As the ice-loaded tags were found to maintain their $5 \mathrm{~m}$ range for 20-50 minutes of thawing, showing that RFID tags are can used to monitor the thawing of ice.

\section{ACKNOWLEDGEMENT}

This work was supported by the UK EPSRC Grant EP/P010164/1. Data will be available from DOI: 10.5258/SOTON/D1849.

\section{REFERENCES}

[1] C. Occhiuzzi, S. Caizzone, and G. Marrocco, "Passive uhf rfid antennas for sensing applications: Principles, methods, and classifcations," IEEE Antennas and Propagation Magazine, vol. 55, no. 6, pp. 14-34, 2013.

[2] G. Marrocco, "The art of UHF RFID antenna design: impedancematching and size-reduction techniques," IEEE Antennas Propag. Magazine, vol. 50, 1, pp. 66 - 79, 2008.

[3] M. Wagih, Y. Wei, A. Komolafe, R. Torah, and S. Beeby, "Reliable UHF Long-Range Textile-Integrated RFID Tag Based on a Compact Flexible Antenna Filament," Sensors, vol. 20 (12), p. 3435, 2020.

[4] R. B. V. B. Simorangkir, Y. Yang, R. M. Hashmi, T. Björninen, K. P. Esselle, and L. Ukkonen, "Polydimethylsiloxane-embedded conductive fabric: Characterization and application for realization of robust passive and active flexible wearable antennas," IEEE Access, vol. 6, pp. 48102 48112,2018

[5] M. Wagih and J. Shi, "Wireless ice detection and monitoring using flexible uhf rfid tags," IEEE Sensors Journal, pp. 1-1, 2021.

[6] R. Kozak, B. D. Wiltshire, M. A. R. Khandoker, K. Golovin, and M. H. Zarifi, "Modified microwave sensor with a patterned ground heater for detection and prevention of ice accumulation," ACS Applied Materials and Interfaces, vol. 12(49), pp. 55 483-55 492, 2020.

[7] A. Carta, A. Ghaghazanian, R. Stefanelli, and D. Trinchero, "Radiofrequency sensors for snow conditions monitoring and real time avalanche alerts," in 2013 IEEE Topical Conference on Wireless Sensors and Sensor Networks (WiSNet), 2013, pp. 49-51.

[8] B. Wiltshire, K. Mirshahidi, K. Golovin, and M. H. Zarifi, "Robust and sensitive frost and ice detection via planar microwave resonator sensor," Sensors and Actuators B: Chemical, vol. 301, p. 126881, 2019.

[9] D. Wang and S. Goldblith, "Dielectric properties of foods," p. 31, 08 1975. 
[10] V. Komarov, S. Wang, and J. Tang, Permittivity and Measurements. American Cancer Society, 2005. 